\title{
Methodology to evaluate glare effects in corrected ammetropic subjects
}

\section{Metodología para evaluar efectos del deslumbramiento en amétropes corregidos}

\author{
Aníbal G. de Paul(1), Elisa M. Colombo(1,2), Silvia. A. Comastri( ${ }^{(2,3, *)}$, Juan A. Aparicio( ${ }^{(4,5)}$, \\ José A. Menéndez ${ }^{(5)}$, Luis A. Issolio ${ }^{(1,2)}$ \\ 1. Departamento de Luminotecnia, Luz y Visión e Instituto de Investigación en Luz, Ambiente y Visión, Universidad \\ Nacional de Tucumán - CONICET, Av. Independencia 1800, San Miguel de Tucumán (4000), Argentina. \\ 2. Consejo Nacional de Investigaciones Científicas y Técnicas, Av. Rivadavia 1917, (C1033AAJ) Buenos Aires, \\ Argentina.
}

3. Grupo de Óptica y Visión, Departamento de Física, Facultad de Ingeniería, Universidad de Buenos Aires, Paseo Colón 850, (C1063ACV) Buenos Aires, Argentina.

4. Departamento de Óptica, Universidad de Valladolid, Paseo de Belén 7, 47071 Valladolid, Spain.

5. Departamento de Estadística e Investigación Operativa, Universidad de Valladolid, P. Preado de la Magdalena s/n, 47071 Valladolid, Spain.

(*) Email: comastri@fi.uba.ar

S: miembro de SEDOPTICA / SEDOPTICA member

Received / Recibido: 23/04/2013. Revised / Revisado: 05/06/2013. Accepted / Aceptado: 10/06/2013.

DOI: $\underline{\text { http://dx.doi.org/10.7149/OPA.46.3.285 }}$

\begin{abstract}
:
Light originated at a glare source and entering the eye, produces a veiling luminance that causes contrast decrease in retinal images and this effect could differ if the subject wears lenses or not. In this work the glare effects in subjects wearing lenses is studied inducing ammetropies in emmetropes. Contrast thresholds of sinusoidal patterns of $2 \mathrm{cpd}$ and $0.5 \mathrm{~cd} / \mathrm{m}^{2}$ are evaluated without and with glare, the glare source being steady, at $12^{\circ}$ and producing $60 \mathrm{~lx}$ at the cornea. To exemplify the use of this methodology, 2 emmetropes under 8 different conditions are considered: naked eye; 3 control conditions (wearing neutral lenses) and 4 ammetropic conditions, inducing low $( \pm 2$ D) and medium $( \pm 5$ D) myopia and hyperopia with positive and negative contact lenses and correcting them with adequate ophthalmic lenses. The contrast threshold differences between the naked eye and the other 7 conditions are statistically not significant without glare and significant (up to more than $100 \%$ ) with glare, independently of lens power and probably due to ectopic scattering and multiple reflections. The method proposed enables the determination of the glare effects if lenses are worn though a greater population is required to attain conclusive data.
\end{abstract}

Key words: Night Driving, Ammetropies, Ophthalmic and Contact Lenses, Contrast Threshold.

\section{RESUMEN:}

La luz originada en una fuente deslumbrante que entra al ojo, produce una luminancia de velo que causa una disminución de contraste en las imágenes retinianas y este efecto podría diferir si el sujeto usa lentes o no. En este trabajo se estudian los efectos del deslumbramiento en sujetos que usan lentes induciendo ametropías en emétropes. Se evalúan contrastes umbrales de patrones sinusoidales de $2 \mathrm{cpd}$ y $0.5 \mathrm{~cd} / \mathrm{m}^{2}$ sin y con deslumbramiento, la fuente deslumbrante siendo estable, a $12^{\circ}$ y produciendo $60 \mathrm{~lx}$ en la córnea. Para ejemplificar el uso de esta metodología, se consideran 2 emétropes en 8 condiciones diferentes: ojo desnudo; 3 condiciones de control (lentes neutras) y 4 condiciones ametrópicas, induciendo miopía e hipermetropía baja ( \pm 2 D) y media $( \pm 5$ D) con lentes de contacto positivas y negativas y corrigiéndolas con adecuadas lentes oftálmicas. Las diferencias de contraste entre el ojo desnudo y las otras 7 condiciones son estadísticamente no significativas sin deslumbramiento y significativas (hasta más de $100 \%$ ) con deslumbramiento, independientemente de la potencia de la lente y probablemente debido a scattering ectópico y reflexiones múltiples. El 
método propuesto permite determinar los efectos de deslumbramiento al usar lentes aunque, para tener datos contundentes, se requiere una mayor población.

Palabras clave: Conducción Nocturna, Ametropías, Lentes Oftálmicas y de Contacto, Contraste.

\section{REFERENCES AND LINKS / REFERENCIAS Y ENLACES}

[1]. J. W. Collins, L. G. Carney, "Visual performance in high myopia”, Curr. Eye Res. 9, 217-223 (1990). DOI

[2]. S. Liou, C. Chiu, "Myopia and contrast sensitivity function", Curr. Eye Res. 22, 81-84 (2001). DOI

[3]. E. Kanonidou, I. Chatziralli, V. Konidaris, C. Kanonidou, L. Papazisis, "A comparative study of visual function of young myopic adults wearing contact lenses vs. spectacles", Contact Lens Anterio. 35, 196198 (2012). DOI

[4]. D. Finlay, J. Wilkinson, "The effects of glare on the contrast sensitivity function", Hum. Factors 26, 283-287 (1984).

[5]. S. Sturgis, D. Osgood, "Effects of glare and background luminance on visual acuity and contrast sensitivity: Implications for driver night vision testing", Hum. Factors 24, 347-360 (1982).

[6]. P. Olson, M. Sivak, "Glare from automobile rear-vision mirrors", Hum. Factors 26, 269-282. (1984).

[7]. T. Schlote, M. Kriegerowski, T. Bende, M. Derse, H. J. Thiel, B. Jean, "Mesopic vision in myopia corrected by photorefractive keratectomy, soft contact lenses, and spectacles", J. Cataract. Refract. Surg. 23, 718-725 (1997). DOI

[8]. D. A. Jewelewicz, R. Evans, R. Chen, S. Trokel, G. J. Florakis, "Evaluation of night vision disturbances in contact lens wearers", CLAO J. 24, 107-110 (1998).

[9]. L. Holladay, "Action of a light-source in the field of view in lowering visibility", J. Opt. Soc. Am. 14, 1-9 (1927). DOI

[10]. J. Vos, "Disability glare - A state of the art report", CIE Journal 3, 39-53 (1984).

[11].J. Vos, B. Cole, H. Bodmann, E. Colombo, T. Takeuchi, T. van den Berg, "CIE equations for disability glare", Commission Internationale d'Eclairage CIE Collection on Glare (2002).

[12]. L. Paulsson, J. Sjöstrand, "Contrast sensitivity in the presence of a glare light. Theoretical concepts and preliminary clinical studies", Invest. Ophth. Vis. Sci. 19, 401-406 (1980).

[13]. M. Abrahamsson, J. Sjöstrand, "Impairment of contrast sensitivity function (CSF) as a measure of disability glare", Invest. Ophth. Vis. Sci. 27, 1131-1136 (1986).

[14]. D. H. Brainard, "The psychophysics toolbox", Spatial Vision 10, 433-436 (1997). DOI

[15].D. G. Pelli, "The VideoToolbox software for visual psychophysics: Transforming numbers into movies", Spatial Vision 10, 437-442 (1997). DOI

[16].M. Kleiner, D. Brainard, D. Pelli, "What's new in Psychtoolbox-3?", Perception 36, ECVP Abstract Supplement (2007)

[17].F. A. Wichmann, N. J. Hill, "The psychometric function: I. Fitting, sampling and goodness-of-fit", Atten. Percept. Psycho. 63, 1293-1313 (2001). DOI

[18].S. A. Comastri, J. Matranga, K. Bastida, L. Issolio, E. Colombo, "Influence of ophthalmic lenses in the illuminance originated at a peripheral glare source and reaching the eye", Optik 120, 860-872 (2009). DOI

[19]. S. A. Comastri, L. Issolio, J. Matranga, E. Colombo, "Influence of ophthalmic lenses in the angle subtended by a peripheral source point", Opt. Pura Apl. 43, 201-206 (2010).

[20]. F. Van Ness, M. Bouman, "Spatial modulation transfer in the human eye", J. Opt. Soc. Am. 57, 401-406 (1967). DOI

[21]. R. S. Longhurst, Geometrical and Physical Optics, Longman, London (1973).

[22]. L. Issolio, N. López Gil, E. Colombo, I. Miró, "Brightness perception under transient glare conditions with myopic observers wearing contact lens and spectacles", Proc. SPIE 4419, 557-560 (2001). DOI

[23].E. Colombo, J. Barraza, L. Issolio, "Effect of a brief exposure on brightness perception in the scotopicmesopic range", Lighting Res. Technol. 32, 65-69 (2000). DOI 
[24]. L. Issolio, P. Barrionuevo, S. A. Comastri, E. Colombo, "Veiling luminance as a descriptor of brightness reduction caused by transient glare", J. Opt. Soc. Am. A 29, 2230-2236 (2012). DOI

[25]. G. C. de Wit, J. E. Coppens, "Straylight of spectacle lenses compared with straylight in the eye", Opt. Vis. Sci. 80, 395-400 (2003). DOI

[26]. A. Tomlinson, G. Mann, "An analysis of visual performance with soft contact lens and spectacle correction", Ophthal. Physiol. Opt. 5, 53-57 (1985). DOI

\section{Introduction}

People with refractive anomalies can correct them by means of spectacles, contact lenses or refractive surgery. In the cases of the non invasive options, the decision of choosing one or the other is usually taken on the basis of medical advice, though other aspects such as comfort and aesthetics are also often considered. Various researchers $[1,2]$ have studied the effects of refractive corrections on subjects with different degrees of myopia comparing contrast sensitivity when using spectacles or contact lenses. In these works, no significant differences were found in groups with low and moderate myopia but, for those with high degrees of myopia, a better performance was found with contact lenses. On the other hand, Kanonidou et al [3] evaluated personal satisfaction provided by both refractive corrections in subjects having moderate myopias and obtained that spectacles were superior to contact lenses.

When the decision concerning which type of refractive correction to be used is taken, its behavior under the presence of glare sources is seldom taken into account. These sources are often present when driving along roads, for instance, when driving at night and encountering an oncoming vehicle with high-beam headlights on or when driving towards the direction of the low sun. In both situations, there is a high loss of visibility [4-6] which can cause serious road accidents. Some researchers have evaluated the influence of the different types of corrections under night vision conditions. For example, Schlote et al [7] measured contrast vision and glare sensitivity under mesopic conditions in myopic eyes corrected by soft contact lenses and spectacles and found that their use does not seem to markedly influence mesopic vision in eyes with low to moderate myopia. Moreover Jewelewicz et al [8] evaluated night vision disturbances for myopic subjects finding that there was no significant difference in image degradation between subjects wearing spectacles and soft contact lenses.

On the other hand, the effect of glare sources in emmetropes wearing no refractive correction is well known. The light generated by these sources and entering the eye is scattered in the ocular media producing a veil on the retina which causes contrast reduction in the retinal images. The equivalent veiling luminance, $L_{v}$, can be determined using the Stiles \& Holladay's equation [9] which is:

$$
L_{v}=\frac{k E}{\theta^{n}},
$$

where the factor $k$ is usually equal to 10 although it varies according to the subject's age and iris color, $E$ is the illuminance generated by the glare source at the cornea, $\theta$ is the angle between the primary visual axis and the glare source position in the visual field, and, according to the CIE $[10,11]$, the exponent $\mathrm{n}$ is usually equal to 2. Moreover, the effect of $L_{v}$ on retinal contrast, $C_{r}$, can be computed using the equation:

$$
C_{r}=\frac{C}{1+\frac{L_{v}}{L_{m}}},
$$

which makes evident the fact that $C_{r}$ is smaller than the stimulus contrast, $C$, and depends both on $L_{v}$ and on $L_{m}$ which is the mean stimulus luminance.

In the present paper we analyze the impairment of mesopic vision caused by glare in subjects wearing contact or ophthalmic lenses of power different or equal to zero. Leaving aside astigmatism and/or other pathologies derived from refractive problems, we evaluate the effects of spherical refractive corrections considering emmetropes on whom we induce different degrees of myopia and hyperopia with positive and negative contact lenses respectively. An advantage of the method we propose is that the analysis can be performed in a controlled 
manner choosing at wish various refraction errors for the same subject (thus leaving aside particular characteristics of different persons). To determine the perceived effect of glare, we measure the contrast threshold of sinusoidal patterns of $2 \mathrm{cpd}$ in the mesopic range of adaptation (mean luminance equal to $0.5 \mathrm{~cd} / \mathrm{m}^{2}$ ) and consider two situations, without and with glare, the steady glare source being located in the periphery of the visual field $\left(\theta=12^{\circ}\right)$. To exemplify the use of this proposed methodology, we consider two subjects though, to fully address the problem, a larger sample of people is required.

\section{Methods}

In what follows we describe the considered subjects and lenses and the methodology we propose.

\section{2.a. Subjects, induced ammetropies and lenses}

The subjects are two young healthy men with normal emmetropic eyes, ADP and PB, 26 and 31 years old respectively, with decimal visual acuity equal to 1.65 in the right eye (which is the evaluated eye). To induce ammetropies, the subjects wear soft contact lenses $C B$ Vision of $-2 \mathrm{D}$ and $-5 \mathrm{D}$ to simulate a low and medium hyperopia and of $+2 \mathrm{D}$ and $+5 \mathrm{D}$ to simulate a low and medium myopia. Each induced ammetropy is concomitantly corrected with an ophthalmic lens yielding the best possible visual acuity (Table I) and taken from a trial lens box (Crown glass; refractive index equal to 1.53; Abbe number around 60; plane internal face). Moreover, we perform control measurements considering, on the one hand, the naked eye (NE) and, on the other, the use of soft Waicon neutral contact lenses (NCL), neutral ophthalmic lenses (NOL) and the combination of both (NCL+NOL) which cause no variation of visual acuities. In the cases with refractive corrections and NCL+NOL, two lenses are worn whereas in the cases NCL and NOL, there is only one lens and this enables the analysis of the influence of each type of lens.

We clean the ophthalmic lenses employing a wet dust cloth to simulate the habitual behavior
TABLE I

Refractive corrections and measured visual acuity

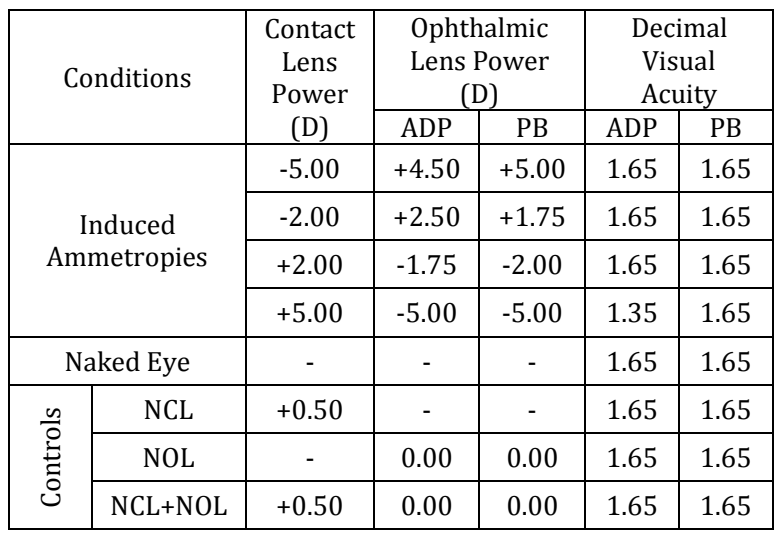

of many lens users instead of washing them with soap and water or alcohol as is customary in optical shops and research laboratories.

\section{2.b. Setup}

The stimuli are achromatic sinusoidal patterns with orientations of +45 and -45 degrees and a spatial frequency of $2 \mathrm{cpd}$ which is a low value also being used by other authors [12,13], presented in a circular patch of 2 degrees in a CRT monitor. We generate these stimuli with functions of the Psychophysics Toolbox library [14-16]. Interposing a neutral density filter between the monitor and the subject, we reduce the monitor luminance to a mesopic level of 0.5 $\mathrm{cd} / \mathrm{m}^{2}$ (measured with a luminancimeter LMT L1009) which is a typical value of night lighting in streets.

In the glare-present situation, we generate glare by the use of an incandescent source subtending $12^{\circ}$ with the visual axis and producing an illuminance at the corneal plane of $60 \mathrm{~lx}\left(L_{v}=4.2 \mathrm{~cd} / \mathrm{m}^{2}\right)$. This source remains on in a steady fashion during the whole experimental session and we take care that the generated light reaches the subject's eye without passing

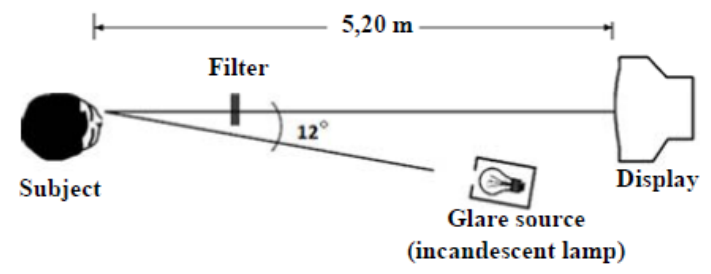

Fig.1. Experimental setup 
through the neutral density filter which regulates the stimulus medium luminance (Fig. 1).

\section{2.c. Procedures and tasks}

Each subject performs two sets of measurements, the first without glare and the second with glare and, in each set, he is examined across 8 experimental conditions: 4 conditions with induced and corrected ammetropies, the naked eye condition and 3 control conditions (Table I). We use a 2AFC method with constant stimuli, the subject's task being to determine the sinusoidal pattern orientation. In each session we examine one refractive correction and measure contrast thresholds presenting 200 stimuli having 5 different contrast levels and presented in a random and balanced way with an exposure time of $200 \mathrm{msec}$. These contrast levels are chosen within a range determined for each subject and glare situation but they are the same for the 8 conditions described above. Before each session the subject is adapted to the monitor mean luminance during 5 minutes, the glare source being turned on in the glare-present situation. A session lasts 20 minutes and each subject performs at most 2 sessions per day, assuring a rest period of at least 2 hours. All measurements are performed with natural pupil and the stimuli are $5.2 \mathrm{~m}$ apart from the subject to eliminate accommodation.

\section{Results}

We obtain contrast thresholds fitting the data to Weibull psychometric functions using a method of maximum-likelihood [17]. In Fig. 2 we depict these contrasts for both subjects, each panel showing both sets of measurements (without and with glare) for the 8 analyzed conditions: the 3 control ones (NCL, NOL and NCL+NOL), the 2 using negative ophthalmic lenses (OL), the naked eye (NE) and the 2 using positive ophthalmic lenses (OL). To facilitate the analysis of results, we indicate the NE level drawing horizontal lines. Error bars are computed by means of a bootstrap analysis grounded on the simulation of a set of Weibull curves based on parameters estimated from the data. A standard deviation with a confidence interval of $95 \%$ is adopted as a criterion.

For the glare-absent situation, the mean pupil diameters (DP) of subjects $\mathrm{PB}$ and ADP are $D_{P B}=5.2 \mathrm{~mm}$ and $D_{A D P}=5.3 \mathrm{~mm}$. For subject $P B$, the contrast threshold is the lowest and equal to 0.055 for the naked eye and increases slightly for the rest of the conditions. For subject ADP, the contrast threshold is 0.048 for the naked eye with slight variations for the rest of the conditions. We compare the contrast threshold for the naked eye to those corresponding to the other conditions, determining $95 \%$ confidence intervals for the differences between the contrasts for the naked eye and for each of the other 7 conditions. For subject $\mathrm{PB}$, the 4 refractive conditions and 2 (NCL and NOL) of the 3 control conditions are not significantly different from the naked eye. For subject ADP, 3 of the 4 refractive conditions (this is, all refractive conditions except $\mathrm{OL}(-5 \mathrm{D})$ ) and 2 (NCL and NCL+NOL) of the 3 control conditions are not significantly different from the naked eye. Hence, taking into account the 7 conditions that correspond to wearing some type of lens, for subject PB, 6 can be considered equal to the

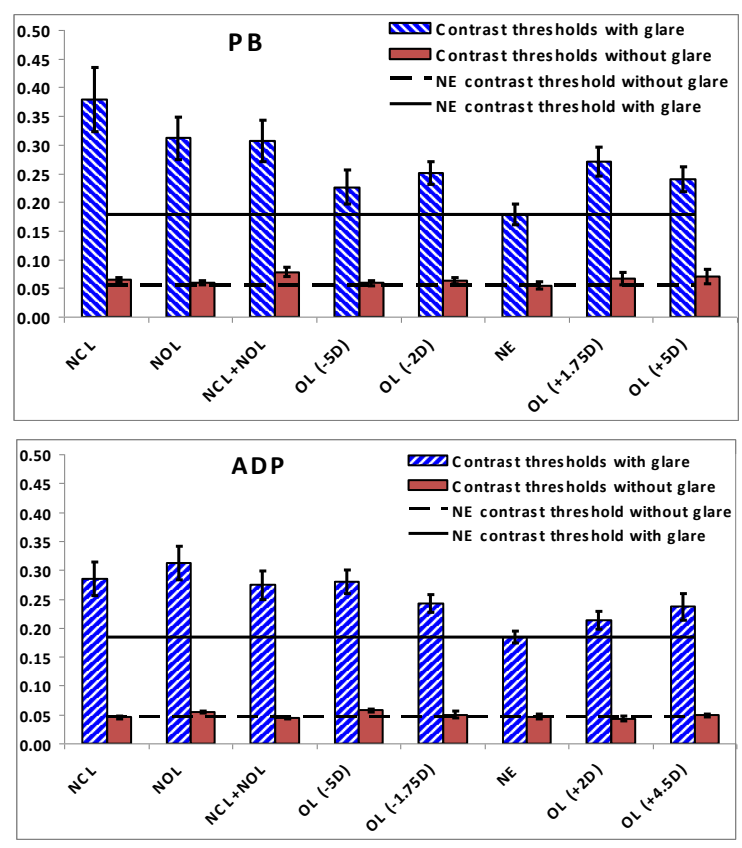

Fig. 2. Contrast thresholds without (plain) and with (stripes) glare determined for the 3 control conditions (NCL, NOL and $\mathrm{NCL}+\mathrm{NOL}$ ), the naked eye (NE) and the 4 refractive correction conditions (OL). The horizontal lines indicate the NE level. Data in each panel correspond to subjects PB and ADP. 
naked eye (only the condition NCL+NOL is significantly different) while for subject ADP, 5 can be considered equal (only the conditions NOL and OL(-5D) are significantly different, the latter coinciding with a visual acuity decrease from 1.65 to 1.35). This suggests that, in the absence of glare, low luminance and low frequency contrast sensitivity in young normal eyes is not affected by the presence or absence of either contact or ophthalmic lenses (with power equal or different from zero) and, also, that for each refractive correction, the compensation between contact lens and ophthalmic lens achieved is usually adequate.

For the glare-present situation, the mean pupil diameters are $\mathrm{DP}_{\mathrm{PB}}=3.1 \mathrm{~mm}$ and $\mathrm{DP}_{\mathrm{ADP}}=3.4$ $\mathrm{mm}$. For both subjects, the smallest contrast thresholds correspond to the naked eye condition and they are 0.178 for $\mathrm{PB}$ and 0.184 for ADP. Determining 95\% confidence intervals for the differences between the threshold contrasts for the naked eye and for each of the other 7 conditions, for both subjects, we obtain that the contrasts for the 3 control conditions significantly differ from that of the naked eye (more than $100 \%$ for the case NCL of PB). Moreover, when comparing the naked eye contrasts to those corresponding to the 4 refractive corrections, results are not uniform for both subjects. For PB we obtain that only the case OL $(-5 D)$ is not significantly different from the naked eye condition while the rest of the refractive conditions yield a significant increase in contrast threshold (approximately 50\% for the case OL(+1.75D)). For ADP we obtain that for both positive corrections (OL $(+2 D)$ and OL(+4.5D)), contrast thresholds are not significantly different from that of the naked eye condition while both negative corrections yield a significant contrast increment (about 50\% for the case OL(-5D)). Thus, taking into account the 7 conditions that correspond to wearing some lens, 6 differ from the naked eye condition for subject PB while 5 differ for subject ADP. In spite of the fact that our results patterns are different across both subjects, our findings suggest that, in the presence of glare, the use of lenses (with or without refractive correction) causes an increase of contrast threshold.

\section{Analysis of results}

The naked eye condition yields the lowest contrast threshold both without and with glare and for subjects PB and ADP we respectively obtain that this threshold is 3 and 4 times greater with glare than without it (Fig. 2), this showing the decrease of visual quality caused by glare. In most cases of the glare-absent situation ( 6 cases for PB and 5 for ADP), there are no significant differences between the naked eye and the other 7 conditions and the few cases of significant differences could be due to psychophysical and/or methodological variations (for example fingerprints in lenses). However, as was to be expected and apparently independently of the lenses power, in the glarepresent situation, the contrast threshold in most of the 7 conditions ( 6 cases for PB and 5 for ADP) is significantly greater than that for the naked eye. This could be accounted for considering that veiling luminance is higher when some lens is worn than for the naked eye and 5 of the potential causes which could contribute in generating this effect are the following:

a) Variations of the glare source illuminance and eccentricity angle due to ophthalmic lenses: Ophthalmic lenses modify the illuminance and eccentricity of the glare source in regards to those encountered for the naked eye, these effects being negligible for contact lenses because vertex distance is almost zero. According to Comastri et al [18], when an ophthalmic lens is worn, the illuminance generated by the glare source that reaches the cornea is influenced by 3 factors which are: pupil size variation of the optical system with and without lens; lateral shifts of rays transmitted through the lens and reflections at the lens. Moreover, considering the lens visual magnification, the eccentricity angle varies in comparison to the angle without lens [19]. Under paraxial approximation, independently of lens shape and considering real-life parameters (pupil diameter, vertex distance, lens axial thickness, refractive index and power, etc.), we $[18,19]$ find that the illuminance and the eccentricity angle increase for a positive lens of power equal or greater than $2 \mathrm{D}$ and decrease 
TABLE II

Percentage of variation of angle, illuminance, veiling luminance and retinal contrast considering $E=60 \mathrm{~lx}, \theta=10^{\circ}$, $4 \mathrm{~mm}$ pupil, $12 \mathrm{~cm}$ vertex distance and $L_{m}=0.5 \mathrm{~cd} / \mathrm{m}^{2}$.

\begin{tabular}{|c|c|c|c|c|}
\hline $\begin{array}{c}\text { Spectacle } \\
\text { Power } \\
(\mathrm{D})\end{array}$ & $\begin{array}{c}\text { Angle } \\
\text { Variation } \\
(\%)\end{array}$ & $\begin{array}{c}\text { Illuminance } \\
\text { Variation } \\
(\%)\end{array}$ & $\begin{array}{c}\text { Variation } \\
\text { of } L_{v} \\
(\%)\end{array}$ & $\begin{array}{c}\text { Variation } \\
\text { of } C_{r} \\
(\%)\end{array}$ \\
\hline+5 & 10.5 & 29.1 & 5.8 & 5.4 \\
\hline+2 & 4.2 & 7.7 & -0.8 & -0.7 \\
\hline 0 & 0.0 & 0.0 & 0.0 & 0.0 \\
\hline-2 & -2.2 & -7.3 & -3.1 & -2.9 \\
\hline-5 & -5.6 & -15.1 & -4.7 & -4.3 \\
\hline
\end{tabular}

for negative lenses. In Table II we consider $E=60 \mathrm{~lx} ; \theta=10^{\circ} ; \mathrm{DP}=4 \mathrm{~mm} ; 12 \mathrm{~mm}$ vertex distance and standard ophthalmic lenses (meniscus type) of power similar to those of the trial box considered in Table I (negative lenses being plane-concave and positive ones planeconvex). In the second and third columns we show the differences in the angle $(\theta)$ and in the illuminance $(E)$ caused by the above mentioned effects. In the fourth column, using Eq. (1), we compute the percentage variation of veiling luminance due to the combination of these variations of $\theta$ and $E$ obtaining that the negative lenses and the $+2 \mathrm{D}$ lens slightly reduce Lv while the positive $+5 \mathrm{D}$ lens increases $\mathrm{Lv}$ in less than $6 \%$. In the fifth column, using Eq. (2) and for $L_{m}=0.5 \mathrm{~cd} / \mathrm{m}^{2}$, we compute the percentage difference of retinal contrast between the cases with and without a lens and obtain that it is always small compared to the variation of Fig. 2 .

b) Modification of the stimulus apparent spatial frequency due to ophthalmic lenses: An ophthalmic lens modifies the size of the retinal image of an object viewed in comparison to the size corresponding to the naked eye, this size being greater for positive lenses and lower for negative ones. Thus a positive lens generates a decrease of apparent spatial frequency in regards to that of the naked eye while a negative lens generates an increase. For the lens powers considered in the present paper, the greatest variation of spatial frequency is lower than $10 \%$, spatial frequency for the naked eye being $2 \mathrm{cpd}$. On the other hand, since adaptation luminance is $0.5 \mathrm{~cd} / \mathrm{m}^{2}$, contrast sensitivity is maximum for a spatial frequency slightly larger than $2 \mathrm{cpd}$ and varies smoothly in its surroundings [20] so a variation of frequency of $10 \%$ causes a small change of contrast threshold which could scarcely influence the results of Fig. 2 and does not account for them.

c) Deterioration of visual performance due to inadequate adaptation of contact lenses: The trademark, materials and base curves of the contact lenses worn by the participants could affect the results and this could be analyzed taking into account the following situations. To adapt contact lenses in clinical practice assuring the optimum patient's satisfaction, rigorous procedures, which are performed during various days and often include controls using topographers, are carried out. On the other hand, in our experiment, the choice of base curves is based on the subjects' feeling of comfort both during and before the trials. Taking into account our results of the glare-absent situation (Fig. 2), the adaptation to contact lenses seems to have a negligible influence in most cases.

d) Multiple reflections at the lenses and at the eye: For the naked eye and considering Le Grand's eye model, a $2.5 \%$ of the light generated at the glare source and reaching the corneal external face is reflected giving rise to the first Purkinje image [21]. On the other hand, though spectacles with anti-reflex coatings reduce multiple reflections yielding better visual quality than standard ones, the lenses we employ have no coating. In the glare-present situation, when contact and/or standard ophthalmic lenses are worn, besides the multiple reflections occurring at their faces, the light reflected at both faces of the cornea and of the crystalline lens returns to each refractive correction, is partially reflected at both faces and returns to the eye. All these glare source images generate illuminances at the cornea which are much smaller than that corresponding to the glare source (the secondary image through a standard spectacle generating approximately 1000 times less illuminance than the primary image). However, these images are located in different places generating veiling luminances which are superposed to that of the primary glare source image and might partially explain the results of Fig. 2.

e) Ectopic scattering in the ophthalmic or/and contact lens: Issolio et al [22] determined the effects of transient glare sources in myopic subjects using either spectacles or contact 
lenses, measuring apparent brightness of a foveal test $[23,24]$. Results showed a brightness reduction both for spectacles and contact lenses when compared to that of the naked eye, though the effects were more pronounced for spectacles. These effects occurred both for subjects with low $(-0.25 \mathrm{D}$ and $-0.75 \mathrm{D})$ and moderate $(-4.25 \mathrm{D})$ myopia; were independent of lens power; were greater than those predicted performing the calculations mentioned in item (a) and were attributed to ectopic scattering. On the other hand, de Wit and Coppens [25] measured the straylight generated by ophthalmic lenses as a function of the angular position along the observation plane (point spread function) and found that this straylight, expressed in terms of $\log S$ ( $S$ being equivalent to the factor $k$ in Eq. (1)), is 10 times lower than that of the naked eye if the lens is clean while its value is similar to that of the naked eye if the lens is dirty because of finger marks. According to these considerations and taking into account that the lenses we employ are cleaned following the usual practice of most lens wearers, scattering in ophthalmic and/or contact lenses could be one of the main causes of the nonuniform results of Fig. 2.

\section{Discussion}

We study the influence of glare on young normal subjects using refractive corrections trying a new methodology based on inducing ammetropies in emmotropes. To do so, we measure contrast sensitivity without and with glare using sinusoidal patterns of $2 \mathrm{cpd}$ under mesopic conditions (mean luminance 0.5 $\mathrm{cd} / \mathrm{m}^{2}$ ). To exemplify the use of our method, we consider 2 young emmetropes (PB and ADP) and 8 refractive conditions for each, 4 correspond to inducing ametropies with contact lenses $( \pm 2 \mathrm{D}$ and $\pm 5 \mathrm{D}$ ) and correcting them with appropriate ophthalmic lenses yielding the best visual acuity, 1 corresponds to the naked eye and 3 correspond to control conditions (neutral contact lens, neutral ophthalmic lens and both lenses).

For naked eyes and for both subjects, we obtain that contrast threshold is 3 to 4 times greater with glare than without it, which shows the decrement of visual quality caused by glare. Moreover, in the glare-absent situation, the use of lenses does not significantly modify contrast sensitivity in most cases $(6$ cases for PB and 5 for ADP). On the other hand, in the glare-present situation, the lowest contrast threshold is attained for the naked eye whereas the use of lenses (independently of their power) produces an increase of contrast threshold between 15\% and more than $100 \%$, which is significant in most cases ( 6 cases for PB and 5 for ADP). The pattern of this increase varies according to the subject, for example, the contrast threshold elevation in the strong myopia condition for PB is smallest than in the other 3 refractive conditions while for ADP it is largest. The nature of the influence of ophthalmic lenses on the resulting patterns is often different from that of contact lenses and various factors might contribute to contrast threshold increase. For instance variations of the glare source illuminance and eccentricity angle due to ophthalmic lenses, modification of the stimulus apparent spatial frequency due to ophthalmic lenses, deterioration of visual performance due to inadequate adaptation of contact lenses, multiple reflections at the lenses and at the eye and ectopic scattering in the ophthalmic or/and contact lens. The superposition of some of these factors could add up to explain the contrast threshold increase found when some lens is worn but, the non-optimum cleanliness of the lenses [25] and the non-uniform results patterns we obtain suggest that one of the main factors is ectopic scattering. To solely analyze the scattering influence, it would be interesting to compare (for fixed external conditions and a given pair contact-ophthalmic lens), the perceptually-based estimates of glare effects in subjects wearing lenses that are carefully cleaned (e.g. using alcohol) and that contain finger marks. Furthermore, though our results for the glare-present situation show a tendency of threshold contrast to increase when wearing contact and/or ophthalmic lenses, there are cases where this increase is not significant and more subjects are required to attain more conclusive data.

On the other hand, our results seem to confirm our previous ones obtained measuring brightness reduction due to the presence of 
glare sources in myopic subjects wearing refractive corrections [22]. Moreover, though in the present paper we do not directly measure the response of myopic and hyperopic subjects wearing either contact or ophthalmic lenses, our results are similar to those of Schlote et al [7] and Jewelewicz et al [8] who did not find important differences in night vision for ammetropic subjects wearing either of the two refractive corrections. Thus, our preliminary results suggest that our methodology of inducing ammetropies in emmetropes could be a reliable way of studying, in a controlled manner, the effects of glare on subjects wearing refractive corrections.

Finally, in the present paper we evaluate visual performance under glare conditions using sinusoidal patterns of low spatial frequency as considered by Paulsson et al [12] and Abrahamsson et al [13]. A similar analysis could be performed using patterns of higher spatial frequency. Taking into account the works of Finlay et al [4] and Tomlinson et al [26], considering medium and high spatial frequencies would enable us to study better subjects' performance when executing visual tasks demanding not only configural processing (low frequencies) but also featural processing (high frequencies).

\section{Acknowledgements}

This research has been done with supports of ANPCyT PICT 2011 №1807, CIUNT 26/E410, and CONICET PIP №0308. Juan Antonio Aparicio thanks the Ministerio de Economía y Competitividad and the Consejería de Educación de Castilla y León under contracts FIS 201122871 and VA005A11-2 respectively. 